Urologe 2020 · 59:602-605

https://doi.org/10.1007/s00120-020-01215-8

Online publiziert: 23. April 2020

(c) Springer Medizin Verlag GmbH, ein Teil von Springer Nature 2020

\section{T. Klotz}

Klinik für Urologie, Andrologie und Kinderurologie, Kliniken Nordoberpfalz AG, Weiden, Deutschland

\section{Subjektive Meinung aus einem „Hotspot"}

Die Corona-Pandemie hat in der BRD mehr oder weniger alle Lebensbereiche erreicht bzw. im Griff. Dies gilt natürlich besonders für die medizinische Versorgung aller Sektoren. Die nachfolgenden Zeilen stellen die subjektive Einzelmeinung eines kommunalpolitisch aktiven Chefarztes dar, der auch in ambulanter KV-Tätigkeit in einer Gemeinschaftspraxis tätig ist.

\section{Beschreibung der Situation - Stand Ende April 2020}

Die Klinik für Urologie, Kinderurologie und Andrologie an den Kliniken Nordoberpfalz AG wird im chefärztlichen Kollegialsystem geführt (Prof. Dr. T. Klotz, Prof. Dr. Dr. Th. Bschleipfer) und stellt eine klassische mittlere Versorgungsklinik mit ca. 2300 stationären Patienten und ca. 4000 ambulanten Patientenkontakten dar. Es wird ein Einzugsgebiet von ca. 250.000 Einwohnern in der nördlichen Oberpfalz in Bayern als urologischer Komplettversorger mit allen Weiterbildungsermächtigungen betreut. Es besteht ein zertifiziertes Prostatakarzinom- und interdisziplinäres onkologisches Zentrum, ein Beckenbodenzentrum und ein reges Studienzentrum. Die Strukturqualität ist weit ausgebaut mit zwei urologischen Arbeitsplätzen, DaVinci, PET-CT, Hybrid$\mathrm{OP}$ etc. Weiterhin ist einer der Chefärzte mit eigenem halben KV-Sitz im Urologischen Zentrum Schwandorf in großer Gemeinschaftspraxis tätig. Damit besteht eine optimale Verzahnung ambulant-stationär mit einem zusätzlich sehr guten
Verhältnis zu den übrigen niedergelassenen Kollegen und der benachbarten Universitätsklinik Regensburg. Soweit so gut.

Im März 2020 wurde unser Einzugsgebiet zum Corona-Hotspot, was durch eine lokale Festivität zu Beginn der Pandemie in einem unserer betreuten Landkreise ausgelöst wurde. Die Infektinzidenz (Landkreise Tirschenreuth/ Neustadt) beträgt weit über 1000 Fälle/100.000 Einwohner und ist damit die Höchste in der BRD. Die Kliniken Nordoberpfalz AG waren damit plötzlich nach den Kliniken um den Kreis Heinsberg in Nordrhein-Westfalen neues Epizentrum in der BRD der stationären Versorgung von Corona-Patienten in ländlicher Umgebung. Glücklicherweise haben wir uns mit überregionaler und lokaler politischer Unterstützung aller Entscheidungsträger und interner sehr kollegialer Absprachen und Ablaufänderungen auf die Versorgung der Patienten optimal und in kurzer Zeit vorbereiten können. Alle zogen/ziehen an einem Strang, sodass wir meiner Meinung nach in sehr überzeugender Weise die Krise medizinisch meistern (konnten). Eine Reihe von Entscheidungsträgern verschiedener Ebenen und v. a. die lokalen Pflegenden haben sich hier meinen uneingeschränkten Respekt, verbunden mit großer Dankbarkeit, erworben.

\section{Organisation in der Krise und Rückblick}

Wie in vielen anderen urologischen Klinken auch wurden auch bei uns alle elek- tiven stationären und ambulanten Eingriffe abbestellt bzw. verschoben. Urologische Assistenten und Pflegepersonal wurden für die Intensivstation und zentrale Notaufnahme abgestellt. Der spezifische urologische Betrieb reduzierte sich bis Anfang Mai 2020auf ca. $20 \%$ des Normalbetriebs. Die wirtschaftliche Situation der Gesamtklinik ist seit Jahren prekär. Dies spielte in der Krise keine Rolle mehr. Allen politischen Entscheidungsträgern aller Ebenen war klar, dass wir uns in einer Ausnahmesituation befinden. Dies ist insoweit bemerkenswert, da noch wenige Wochen zuvor das abstruse MDKReformgesetz und eine Verkürzung der mittleren Verweildauer um 0,4 Tage als „segensbringend“ auf Strategietagungen neben der Möglichkeit der Insolvenz diskutiert wurde. Damit ergab sich von meiner Seite plötzlich eine völlig neue Sicht auf viele Dinge, die noch wenige Wochen zuvor als sehr bedeutsam erschienen.

\section{Die Urologie ist wichtig - aber sehr vulnerabel!}

Unser Fach hat sich in den letzten Jahrzehnten hochspezialisiert zum Wohle der Patienten entwickelt - daran besteht für mich kein Zweifel. Allerdings wird in der aktuellen Ausnahmesituation schnell klar, dass hierin auch eine hohe Vulnerabilität in Krisensituationen besteht. Das gilt natürlich auch für andere Fachgebiete, aber für die Urologie besonders und z. B. für die Neurologie oder Kardiologie weniger. Die Wertigkeit bezüglich der Patientenbehandlung innerhalb eines großen Versorgungs- 
hauses hatte sich rasch verschoben. Dies liegt u.a. daran, dass viele der urologischen Krankheitsbilder nur eingeschränkt zeitkritisch sind. Natürlich muss ein aggressives Prostatakarzinom oder ein invasiver Blasentumor rasch einer Behandlung zugeführt werden hier ist insbesondere von der DGU eine hervorragende Priorisierungsliste schon im März 2020 herausgegeben worden - aber ein akuter Herzinfarkt, Apoplex, Oberschenkelfraktur oder eine intensivpflichtige COVID-Pneumonie sind zeitkritischer. Das bedeutete in unserem Fall, dass sich die urologische Tätigkeit sehr stark auf Ableitungen (DJ etc.) und kleine bis mittlere Operationen (Pyonephrosen, Koliken, Hämaturien, Torsion etc.) fokussiert hat. Natürlich wurden auch noch einzelne drängende Tumoreingriffe durchgeführt, aber nicht in vergleichbarer Häufigkeit wie vor der Krise. Für einen älteren spezialisierten operativen Urologen war dies eine schmerzliche Erfahrung. Die eigene Wichtigkeit in der Klinik als langjähriger etablierter Chefarzt hat sich auf einmal relativiert und trat hinter der des Anästhesisten und Intensivmediziners zurück.

Auch in der KV-Praxis änderte sich viel. Hier hat sich das Spektrum der Krankheitsbilder verschoben - Nachsorgen, Testalgien, ED, PSA-Testung relativieren sich ebenfalls. Ein volles Wartezimmer steht nicht mehr für gute Urologie, sondern für hohes Risiko. Ein Wechsel von suprapubischen Kathetern im Altenheim bei einem möglichen COVID-Patienten gewinnt auf einmal ein ganz anderes Organisations- und Problemniveau. Die Patientenzahl insgesamt hat aktuell in der KV-Praxis deutlich abgenommen. Was das für die Zukunft einiger Praxen bedeuten mag, kann ich nicht sagen - aber es ist sicher nicht positiv unter dem derzeitigen Finanzierungssystem.

\section{Relativierung urologischer Krankheitsbilder}

In vielen Bereichen der Urologie gibt es Krankheitsbilder, über deren Behandlungsintensität in Abhängigkeit von der individuellen Patientensituation trefflich diskutiert werden kann. Hier möchte ich onkologisch das Low-risk-Prostatakarzinom, den kleinen Nierentumor oder den oberflächlichen Harnblasentumor erwähnen. Im nicht-onkologischen Bereich sind der asymptomatische Kelchstein, die kompensierte Ureterabgangsstenose oder die IPP Krankheitsbilder von sekundärer Relevanz. Natürlich gibt es für die meisten Stadien und Situationen hilfreiche Leitlinien mit guter Evidenz. Aber ist die Wertigkeit für die medizinische Versorgung wirklich so hoch? Die Aufzählung ließe sich natürlich auch für viele nichturologische Erkrankungsbilder beliebig fortsetzen. Dennoch zeigte sich für mich, dass wir in den letzten Jahren eine z.T. akademische Diskussion geführt haben, die wenig nutzbringend war. Eine Krise wie diese entlarvt ganze Kongresssitzungen als nährwertarm, auch wenn ich das keinesfalls als Vorwurf verstanden haben will. Ich denke jetzt über einige Krankheitsbilder anders als vor der Krise und ich bin mir sicher, dass es nicht nur mir so geht.

\section{Subjektive Einschätzungen zur Zukunft der Urologie?}

Ich bin überzeugt, dass die Corona-Pandemie uns über Jahre sowohl von Seiten der medizinischen Versorgung als auch von Seiten der Finanzierung beschäftigen wird. Einen Status wie vor der Krise kann es nicht geben, allein deshalb nicht, weil die ganzen Rettungsschirme auch außerhalb des Gesundheitswesens die Grenzen unserer Leistungsfähigkeit brutal ausloten werden. Die Frage nach der Ressourcenallokation wird früher oder später in die tatsächliche Versorgung durchschlagen, aber anders als wir bisher dachten. Es geht nicht mehr um Zentralisierung, Ökonomisierung und Spezialisierung der Medizin, sondern um hochwertige Grundversorgung auf noch zu bestimmender Basis. Aktuell ist die medizinische Notfallversorgung im absoluten Fokus aller Diskussionen, in 12 Monaten wird sich dies in Richtung gesamtwirtschaftlicher Ökonomie und systemrelevanter Grundversorgung im weiteren Sinne verschieben.
Was könnte dies für die Urologie bedeuten? Hier möchte ich einige Überlegungen anstellen, die wahrscheinlich nicht unwidersprochen bleiben werden. Ein 80-jähriger fitter Patient mit einem nicht-metastasierten kastrationsrefraktären oder einem primär metastasierten Prostatakarzinom sollte aktuell eine medikamentöse Tumortherapie mit Monatstherapiekosten von ca. $5000 €$ und bei Ansprechen für hoffentlich mehrere Jahre bekommen. Dies ist medizinisch völlig klar und mit hoher Evidenz abgesichert, aber in der zu erwartenden Ressourcendiskussion sehr komplex zu sehen. Wir dürfen weiterhin nicht vergessen, dass dieses Bespiel für eine Vielzahl von Erkrankungen auch in anderen Fachgebieten gilt - mit anderen Worten, die bisherige ökonomisch getriggerte Diskussion mit dem Deckmäntelchen der Qualitätsverbesserung, die über GBA, Zertifizierung, Mindestmengen, Onkologievereinbarung weitgehend im medizinischen Kontext mit harten Bandagen geführt wurde, wird nun um eine noch schwierigere ethische Diskussion erweitert. Ich sehe uns hier in der BRD keinesfalls gewappnet, da es zwar schon lange eine ethische Versorgungsdiskussion gibt, die aber nur als Feigenblatt zur Profilierung einzelner Akteure und mehr oder weniger akademisch geführt wird.

Ähnliches gilt für einige OP-Verfahren, was uns technikverliebte Urologen schmerzen wird. Eine Robotik, die nur an wenigen Klinken kostendeckend ist, wird möglicherweise wieder zurückgefahren, zumal der Marketingeffekt immer mehr an Bedeutung verliert und es nur eine überschaubare Evidenz gibt, dass die robotischen Verfahren besser sind.

Jeder kann für seine Klinik und sein Umfeld selbst beurteilen, welche Auswirkungen möglich sind. Für mich wird klar, dass die Hochspezialisierung, wie sie an einigen Häusern und Ambulanzen stringent erfolgte, nun zum Risiko wird und eine breite urologische Basisversorgung an Wert gewinnt.

Das fallzahlorientierte DRG-System und auch das budgetierte KV-System haben meiner Meinung nach keine $\mathrm{Zu}$ kunft mehr - aus Sicht eines älteren Klinikers „Gott sei Dank“. Die Zertifizie- 
rungswut, das gegenseitige Hochrüsten von MDK und Klinikabrechnungswesen, die „Fokuslistenonanie“ vieler Kollegen, die verbittert geführt wurde, um sich einen Vorteil gegenüber den Konkurrenten des eigenen Fachs zu schaffen, war von betriebswirtschaftlicher Seite gefördert und verständlich, aber in Wirklichkeit eher peinlich. Der jährliche Stress und Aufwand für die Abarbeitung von Erhebungsbögen für die Zertifizierung, allein um des gerahmten Zertifikats willen und um Zertifizierungsfirmen großzügig alimentieren und Auditoren zu hofieren, war schon lange unerträglich ich hoffe, dass hier Vernunft einkehrt. Die Chancen stehen hierfür jetzt gut. Ähnliches gilt in der KV-Praxis - unrealistische Hygieneanforderungen, Regressandrohungen, wechselnde Rabattverträge und Budgetierung, das pervertierte Bestehen der Kostenträger auf die Einhaltung von Formalien müssen ein Ende haben.

\section{Assistenzberufe und Urologie}

Klar wird, dass unsere Assistenzberufe in Praxis uns Klinik unersetzlich sind und bisher krass unterbezahlt waren. Der Personalmangel hat dies die letzten Jahre bereits aufgezeigt, aber die aktuelle Krise betont nochmals auch von Seiten der Bedeutung und erforderlichen Wertschätzung die Arbeit der Mitarbeiter. Ohne Assistenzberufe und urologische Pflege gibt es keine vernünftige Urologie. In den Klinken wurde die Pflege über Jahre vernachlässigt und nur als Kostenfaktor gesehen. Ich kann mich an Tage erinnern, an denen zwei Schwestern 25 Patienten versorgt haben, von denen 10 dement waren. Das kann nicht so bleiben. Hier besteht jedoch, so glaube ich, ein sehr breiter Konsens, den auch Kostenträger, Geschäftsführer und Gesundheitsökonomen akzeptieren müssen.

\section{Zahl der Kliniken in der BRD und Zentralisierung}

Noch vor weniger als einem Jahr tönte die Bertelsmann-Stiftung, dass mindestens ein Drittel der Kliniken geschlossen werden muss. Kostenträger, Politik und viele Insider sahen das genauso. Nur die belächelten Regionalfürsten wollten ihr Krankenhaus weiter behalten und haben mit Fallzahlsteigerung, Verbünden, Zuschüssen und Spezialisierung gegen die Zentralisierung in Großkliniken gekämpft. Ich muss zugeben, dass auch ich der Idee der Überversorgung in weiten Bereichen nachhing und viele kleinere Kliniken für überflüssig hielt. Ähnliches mag vielleicht der eine oder andere Universitätsordinarius auch für Kliniken unserer Größe gedacht haben. Wie oft musste man lesen, dass die eine oder andere Prozedur nur an einem spezialisierten Zentrum (meist universitär) stattfinden sollte. Es ist der Schlusssatz im Abstract fast jeder klinischen Arbeit und, ehrlich gesagt, schon immer ein Zeichen von peinlicher Arroganz.

Diese Diskussion hat sich erledigt. Das lokale Krankenhaus wird als Grundversorger mit Teilspezialisierung bestehen bleiben. Ohne diese Häuser hätten wir die Krise nicht meistern können. Die gelobten hocheffizienten zentralisierten Systeme in anderen Ländern mit Riesenambulanzen, kurzer Liegedauer und tollen Ergebnissen haben sich als unkalkulierbares Risiko gezeigt. Die Bereitschaft junger Chefärzte, Geschäftsführern mit immer kürzerer Liegezeit und höheren Anzahl von Prozeduren hinterherzuhecheln und dies in Hochglanzbroschüren und auf Kongressen mit stolzer Brust auch noch als besondere Qualität zu propagieren, wird abnehmen. Bedeutet aber auch, dass für viele chefärztliche Kollegen/innen das Einkommen sinken wird.

Die stationäre heimatnahe Versorgung wird nun als kostenintensive $\mathrm{Da}$ seinsvorsorge akzeptiert, was eigentlich bei einer überalternden Bevölkerung auch richtig ist. Natürlich muss es eine höherspezialisierte Versorgung auch weiter geben, aber die Ressourcenallokation wird sich mit dem Finanzierungssystem ändern. Die Diskussion, wer welchen Anteil an Ressourcen erhält, wird nicht erfreulich, da auch in der Urologie die Existenz ganzer Geschäftsmodelle nun in Frage gestellt ist.

\section{Zusammenfassung}

Das Gesundheitssystem wird sich in der BRD dramatisch verändern. Der gesellschaftliche Fokus rückt von der Wirtschaftlichkeit in effizienten Strukturen zur Daseinsvorsorge und Systemrelevanz. Die pseudowirtschaftlichen DRG- und KV-Systeme werden sich reformieren müssen, was nicht bedeutet, dass es sich zum Besseren wendet. Es bleibt zu hoffen, dass die marketinggesteuerte "Zentritis“ sich relativiert. Die Urologie mit ihrer Hochspezialisierung wird sich einen neuen Platz im Fächerkanon erkämpfen müssen, wobei die evidenzbasierte Diskussion unter Fachspezialisten um eine sehr schwierige ethische Diskussion erweitert wird. Der Ausgang ist hier völlig offen. Ein unbedingt positiver Aspekt ist jedoch $\mathrm{zu}$ betonen: DGU und BvDU werden notgedrungen zusammenrücken. Die Zeit der Befindlichkeiten und Erbhöfe ist nun endgültig vorbei.

\section{Korrespondenzadresse}

Prof. Dr. T. Klotz, MPH

Klinik für Urologie, Andrologie und

Kinderurologie, Kliniken Nordoberpfalz AG

Söllnerstr. 16, 92637 Weiden, Deutschland

Theodor.klotz@kliniken-nordoberpfalz.ag

Interessenkonflikt. T. Klotz gibt an, dass kein Interessenkonflikt besteht. 
Hier steht eine Anzeige.

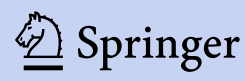

